\title{
New College English Translation Skills of English and Chinese
}

\section{Comparative Perspective}

\author{
Yi Liu \\ School of Foreign Studies,Anhui Sanlian University, Hefei 230601,China
}

\begin{abstract}
Keywords: English Contrast; English translation; Translation skills
\end{abstract}
\begin{abstract}
In college English teaching, translation teaching has always been a weak link lies, many students do not have a solid foundation in English translation, was very difficult during the course of the study. This had a negative impact on their overall level of English improve. In this paper, English Contrast Perspective, by analyzing the current situation and common errors of translation, made several university English translation skills, as used in teaching and have achieved good results.
\end{abstract}

\section{Introduction}

With the diversification of economic exchanges and cultural globalization, the economic, political, cultural and other aspects of the increasingly strengthened, the social demand for translation professionals is also increasing, those who not only professional and good at English complex talent is becoming scarce talent. Current college CET conform to changes in social demand for talent. From December 2013, CET Questions section for translation made a new adjustment, the original single sentence translation to translate a paragraph, the proportion of its score from 5 percent to 15 percent, exam time is 5 minutes from the adjustment 30 minutes. The original single sentence translation mainly assess students ability to use the correct vocabulary and grammatical structures. And now consists of a single sentence translation to translate a paragraph, clearly improve the ability of students to translate requirements. But for now our domestic translation techniques used and the ability to translate aspects of college students there are still some deficiencies. These are often the lack of Chinese and Western culture and a different result, it is now more common myths Chinglish. Throughout recent years, college English translation teaching, is also true. College English translation teaching college English teaching has always been a weak link, college English translation capability has been not optimistic. Zhangmei Ping, Liu Xiaomin study found that many students to translate foundation is not solid, lack of basic translation skills and techniques. ${ }^{[1]}$ In translation practice, many students lack awareness of the difference between Chinese and English languages, the translation of "Chinglish" or "Chinese Europeanization" is widespread.

On the role of mother tongue in foreign language teaching, language in the world it has been controversial. Some scholars believe that it should strengthen the role of mother tongue in foreign language learning, and the appropriate use of mother tongue and foreign language teaching as a way of comparison. Mr. Lv Shuxiang famous linguist that is representative of this view, he once said: "I believe that Chinese students are most useful to help him recognize the differences between English and Chinese, in each specific question - form, meaning and grammatical categories, sentence structure, as far as possible with the case of Chinese and English, let him get through this comparison a deeper understanding. " ${ }^{[2]}$ in contrast, native English speaking students in learning Chinese, as well. This view full recognition of the mother tongue has a positive role in the migration of foreign language learning, students learning a foreign language using the similarities of 
the two languages, by comparison, is easier to learn a language, to promote foreign language learning plays role.

To be a very good improvement "Chinglish" or "Chinese Europeanization" of this phenomenon, to improve the quality of English translation, translation teaching theories and methods of introducing English Contrast is very necessary translation skills taught in English Contrast perspective can enhance translation targeted and systematic teaching, teaching to achieve a multiplier effect.

\section{The current situation of college English translation}

\section{(1) College English Translation Course enough emphasis}

Most courses in English professional translation of domestic institutions only 2 hours, non-English majors are more scarce, translation teaching materials are outdated and simplistic, lack of practicality, the teachers and students generally pay more attention to listening, speaking, reading and writing in English in the traditional sense of these basic skills, while ignoring the best embodies the goal that is communication in foreign language teaching translation skills.

\section{(2) Teaching philosophy and teaching methods lack of innovation}

Translation Teaching of English teaching in long fringes, translation teaching theories and methods of the lack of systematic, practical, translation teaching model focuses on the words, syntax and idioms to explain, too single teaching method, students lack a coherent way of thinking Textual Cohesion in Translation lack of opportunity to practice exercises translation theory and practical application of a combination of students had their tables may not its essence. Therefore, translators should pay attention to teaching receptive skills and productive skills training, focusing on the relationship between language input and output, pay attention to grasp the relationship between culture and language knowledge translation capabilities.

(3) Students' Intercultural Communication Competence and awareness need to be strengthened

Translation is a language and cultural significance of carrying conversion to another language and culture in cross-language, cross-cultural communication, language is the carrier of culture, language is the mirror of culture. ${ }^{[3]}$ translation itself is a process of verbal communication, which involves not only the language code, the more important is the dynamic context based dynamic reasoning, and reasoning is based on the relevance of translation theory and pragmatics combination how to deal with cultural differences when it comes to the correct translation. Only a profound understanding of cultural difference, students cross-cultural communication skills and awareness, in order to make sense of language translation is accurate, culturally appropriate sense, we can really translate the authentic fluent translation.

\section{The common cause of the error analysis}

\section{(1) Differences of cultural background knowledge}

Translation is the significance of a language expressed expressed with another language cultural activities. ${ }^{[4]}$ Thus, the importance of the role of cultural background knowledge in Translation imagined. Differences in cultural background is one of the main causes of translation errors. Only in accordance with the literal meaning of the word to translate, regardless of differences in background knowledge of Eastern and Western cultures, will be sudden, a lot of jokes. For example: "the black sheep", if you are willing to follow refers to the "black sheep", in fact, the English language is a "black sheep" means. In the process of Translation, the language is the basis 
of cultural knowledge is the key. Therefore, translation should give more consideration to differences in cultural background knowledge, so as to avoid the occurrence of errors.

\section{(2) Out of context, the words too literally}

If the translation from the specific context, we put this phenomenon is called out of context. Lack of contextual analysis of the wording, it will inevitably make mistakes too literally. So before we start writing, the first thing to do is to deeply understand the original meaning, only in this way, when the translation will not commit out of context, the words too literally errors. Like "This was the last straw", we can be translated as "This is the last straw", but this sentence on the specific context actually translated into "a series of combat," two are quite different meaning.

\section{(3) The original meaning Sikou dictionary}

Some people think that as long as the dictionary translation is not a difficult task, but they do not know the meaning of English words is more flexible, a word may have several different meanings, if only in accordance with the dictionary meaning of words to rote error the inevitable. Translation, sometimes a word means not only to link this phrase means, but also the concept of full integration, considering the job.

\section{(4) Can not increase or decrease the word word}

It requires translation is faithful to the original, the original text content can not make any additions or subtractions, but because of the English and Chinese languages belong to two different languages, there are many differences in wording sentences. If we just blindly according to the original translator, translated articles will be very strange. Therefore, we in the translation of the article, should be properly supplement when needed or delete some words to make the translation smoother, more consistent with the expression language.

\section{Under English and Comparative Perspective common translation skills}

Chinese and English language differences in vocabulary, semantics, syntax, pragmatics, determine the two languages in the conversion can not be completely equivalent translation, depending on the context requires a different translation skills. The following discusses some common translation skills from English Contrast perspective.

\section{(1) Transfer of translation}

Chinese and English in their development in the formation of a static dynamic characteristics of Chinese and English, the Chinese use more verbs and English verbs tend to use parts of speech other than expression. Rhetoric is the essence of English static nouns and prepositions advantages advantages, and the advantages of the preposition is the inevitable result of the advantages of a noun. Due to the aid between nouns and noun preposition junction. ${ }^{[5]}$ Therefore, in the Chinese translation often becomes "action" is "static", to get rid of a large number of verbs interference, static color highlighting. Chinese-English translation, the transfer of translation is often used in translation skills. The so-called sexual revolution is the translation in the translation process, in accordance with customary language translation of part of speech conversion, such as: the original text of a noun into a verb in the original text is converted to adverbs prepositions.

(i) Lin believes that a successful ban of the opium trade, you have to first destroy opium.

Translation: Lin Zexu believed that a successful ban of the trade in opium must be preceded by the destruction of the drug itself.

(ii)That guy always endless talk incessantly.

Translation: That fellow is very talkative. 


\section{(iii)Take the subway to work.}

Translation: He usually rides subways to and from work.

Examples of the above three verbs are converted to the corresponding noun, adjective and preposition forms of expression, the translation is simple, authentic, consistent with the English expression habit. As can be seen from the above cases of transfer of translation, translation Word Conversion existence of certain laws, the most obvious point is that English-speaking people prefer to use nouns and prepositions, while Chinese verbs with more than a number. Thus, in the Chinese-English translation, as the case may be, we can Chinese verb or verb phrase is converted to English noun, prepositional phrase, etc. in order to conform to the characteristics of English.

\section{(2) Increase translation}

Add translation refers to the translation by the semantics, syntax or rhetoric needs, based on the original add the necessary words, phrases, etc. ${ }^{[6]}$ so that the translation of the formal grammar and language translation in line with the habits and cultural background, the words Lenovo side into line with the original, so that in the translation and the original content, form and spiritual aspects of peer to peer up.

(v) Their wives quarrel, unfortunately I caught.

Translation: I chanced to be present when the sisters - in - law were having a quarrel.

\section{(vi) July 28, 1976, Tangshan earthquake happened.}

Translation: On July 28, 1976, a big earthquake took place in Tangshan.

Chinese sentences as "water syntax", less or even no conjunctions wording remains smooth, while the English are "bamboo syntax", English sentence is the interface between the various conjunctions made of bamboo as steadily linked. The most important difference between the Chinese and English language feature is that the meaning and hypotaxis distinction. ${ }^{[7]}$ Example (4) by adding conjunctions reflect time when relations between the two clauses.

\section{(3) Change order Translation}

Because there is a big difference between Chinese and English on the grammatical structures and habits of expression, in the translation process, according to the expressions of the target language is used to adjust the original word order, a part of the upcoming original sentence (words, phrases, phrases, clauses, ) position changes to make it more in line with the target language sentence translated language habits, so that the maximum sentence translated language fluent.

\section{Application of contrast effect}

The author in order to better detect differences between Chinese and English, took control experiment method to solve the problem. Experimental group and control group were two experimental classes a middle school. Specific data obtained after completion of the experiment shown in Table 1. Through the analysis of the data in Table 1 shows that in college English translation teaching of English and Chinese perspective, increase student transfer method of translation, adding French translation, French translation and other translation skills are omitted, can effectively promote students' English enhance translation capabilities.

Table 1 before and after the experiment two classes Translation compare the average

\begin{tabular}{l|c|cc}
\multicolumn{3}{c}{ score (points) } \\
\hline \multirow{2}{*}{ Group } & Number of people & Before the & English average \\
\cline { 3 - 4 } & & experiment & experiment \\
Class 1 & 42 & 66.3 & 77.1 \\
Class 2 & 45 & 61.5 & 70.4 \\
\hline
\end{tabular}




\section{Conclusion}

University English translation Is the Chinese and English languages on the complex process of transformation in thinking and language, in English Learning and our everyday communication is increasingly important. College English Translation Teaching to let students understand the concept of translation as well as the standard translation, then through their own accumulation of knowledge, through specific practices to improve their translation skills. Throughout the translation process, we always want to notice a difference between the two, and the difference between the two is reflected in the specific process. ${ }^{[8]}$ translation should fully take into account differences between Chinese and Western cultural background knowledge, accurate and smooth for translation activities.

The introduction of university courses in translation in contrast to the concept of teaching, so that everyone understands the difference between Western expression in contrast to the process, the final translation so that we enhance the capacity, in line with market demand. College English Translation Teaching English Contrast introduced the theory and method of teaching is an effective way to improve translation quality. Taught in English Contrast Perspective translation skills, will help improve students' awareness of the differences in the Chinese-English translation practice, translation skills to enhance understanding and application, improve translation capabilities.

\section{Acknowledgement}

Author Brief Introduction: Liu Yi,(1989-), Male, Anhui Hefei, Bachelor Degree, Teaching Assistant, Research Direction: Oral English and Translation.

Fund Project: This paper is the research result that focused on humanities and social sciences key project of Anhui Provincial Education Department “A Comparative Study of Faulkner and Mo Yan works of modern and post-modern Theory of overall under” (Item Number: SK2014A245).

\section{References}

[1] Ma Jingfeng. Student-oriented skills-oriented activation Translation Practice Teaching [J]. Industry and Science \& Technology Forum 2016 (11).

[2] Lü. Chinese people learn English [M]. Commercial Press, 1980.

[3] Jiang winter and Translation Studies Cultural proprietary menu items - the "cultural turn" Perspective [J] Guangdong Polytechnic Normal University 2014 (06).

[4] Sun Yifeng. Translation and Intercultural Communication Strategies [J]. Chinese translation. $2012(01)$.

[5] Zhang $\mathrm{Li}, \mathrm{Xu}$ silver. Construction Grammar for Chinese "adjective + object" structure of research [J]. Foreign Languages (Shanghai International Studies University). 2011 (06).

[6] Pan Yueming, Guoxiu Zhi Publicity Translation Strategies of colleges and universities Zhejiang University of Technology in school history for translation [J] Chinese Science \& Technology Translators 2011 (04).

[7] Guo strong. Chinese English Parataxis shaped to reflect the comparative study together [J]. Yunan Normal University. 2005 (05).

[8] Zhoufa Xiang eds. New perspectives on international translation [M]. Baihua Literature and Art Publishing House, 2006. 